\title{
Meningococcal Antigen Typing System Development and Application to the Evaluation of Effectiveness of Meningococcal $B$ Vaccine and Possible Use for Other Purposes
}

\author{
Alexander Domnich, ${ }^{1}$ Roberto Gasparini, ${ }^{1}$ Daniela Amicizia, ${ }^{1}$ Giuseppe Boccadifuoco, ${ }^{2}$ \\ Marzia Monica Giuliani, ${ }^{2}$ and Donatella Panatto ${ }^{1}$ \\ ${ }^{1}$ Department of Health Sciences, University of Genoa, Via Pastore 1, 16132 Genoa, Italy \\ ${ }^{2}$ Novartis Vaccines, Via Fiorentina 1, 53100 Siena, Italy \\ Correspondence should be addressed to Donatella Panatto; panatto@unige.it
}

Received 24 November 2014; Revised 16 January 2015; Accepted 17 February 2015

Academic Editor: Saul N. Faust

Copyright (C) 2015 Alexander Domnich et al. This is an open access article distributed under the Creative Commons Attribution License, which permits unrestricted use, distribution, and reproduction in any medium, provided the original work is properly cited.

\begin{abstract}
Development of the 4-component meningococcal serogroup B vaccine (4CMenB) has required new assays for the reliable evaluation of the expression and cross-reactivity of those specific antigen variants that are predicted to be targeted by bactericidal antibodies elicited by the vaccine in different isolates. Existing laboratory techniques, such as multilocus sequence typing, are poorly suited to this purpose, since they do not provide information on the contribution of single vaccine components and therefore cannot be applied to estimate the potential coverage of the multicomponent vaccine. The hSBA, the only correlate of protection against invasive meningococcal disease accepted thus far, cannot conveniently be used to test large number of strains. To overcome these issues, the meningococcal antigen typing system (MATS) has been specifically developed in order to predict 4CMenB coverage of individual meningococcus serogroup B strains. To date, MATS has proved advantageous for several reasons, including its ability to assess both qualitative and quantitative aspects of surface antigens of single strains in a highly reproducible, rapid, and resource-saving manner, while its shortcomings include a possible underestimation of $4 \mathrm{CMenB}$ coverage and the use of pooled sera to calculate the positive bactericidal threshold. This paper provides an overview of MATS development and its field application.
\end{abstract}

\section{Introduction}

Neisseria meningitidis is a major causative agent of invasive bacterial diseases that affect mostly children between 3 and 12 months of age, followed by adolescents. Of thirteen known serogroups of N. meningitidis, only six (A, B, C, W-135, X, and $\mathrm{Y}$ ) cause invasive disease $[1,2]$. Active immunization is the most effective way to prevent invasive meningococcal disease; vaccines against serogroups $\mathrm{A}, \mathrm{C}, \mathrm{W}-135$, and $\mathrm{Y}$ and a recently approved universal vaccine against serogroup B (MenB) are available [3]. This latter, a 4-component meningococcal serogroup $B$ vaccine (4CMenB, commercially available as Bexsero), is the first vaccine to be developed by means of reverse vaccinology $[4,5]$. $4 \mathrm{CMenB}$ consists of three recombinant proteins, namely, factor $\mathrm{H}$ binding protein ( $\mathrm{fHbp}$ ), Neisserial heparin-binding antigen (NHBA), and Neisseria adhesin A (NadA), combined with OMV from MenB strain NZ98/254, which contains porin A (PorA) serosubtype P1.4 (see Figure 1) [6].

Evaluating the protective efficacy of a vaccine without measuring clinical outcomes is of great practical importance $[7,8]$. This is particularly true for vaccines against $N$. meningitidis, the incidence of which is relatively low $[9,10]$. A correlate or surrogate of protection can be defined as an immunological measurement that correlates statistically with the level of a trial endpoint used to measure vaccine efficacy [11]. The serum bactericidal assay with human complement (hSBA) is a universally accepted correlate of protection against meningococcal disease that quantifies the complement-mediated killing of bacteria by functional antibodies in sera from vaccinees [12]; in general, an hSBA titer $\geq 1: 4$ is considered to be a correlate of protection $[12,13]$. 


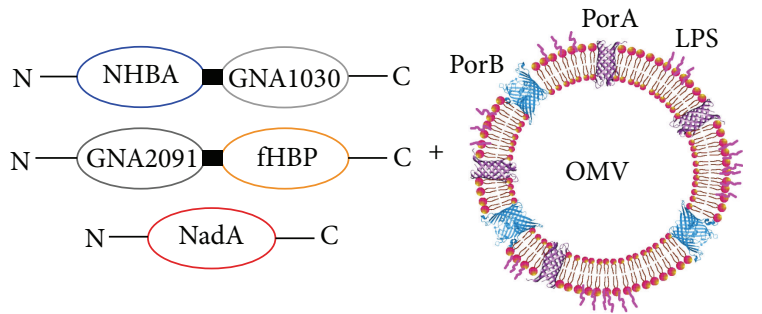

FIgURE 1: 4CMenB vaccine composition. Antigens NHBA and fHbp are fused with two accessory proteins, GNA1030 and GNA2091, respectively. Adapted with permission from [15].

Despite its strengths, hSBA has some shortcomings. First, hSBA is a labor-intensive technique and testing a large number of single circulating strains would produce logistical difficulties. Second, it requires collecting considerable amounts of sera from immunized individuals, which would be ethically debatable, especially in pediatric studies. Third, the standardization of hSBA across numerous strains and complement sources is also burdensome [5, 14, 15]. Fourth, while hSBA is able to assess the effectiveness of a vaccine by measuring bactericidal antibody titers, it does not provide information on the contribution of each vaccine component [14]. Indeed, the surface-exposed proteins $\mathrm{fHbp}, \mathrm{NHBA}$, and NadA of MenB display considerable sequence variation and expression, as well as different degrees of cross-reactivity among variants of a protein antigen to the antibodies induced by the vaccine [16-20]. We therefore need new assays that can reliably assess the expression of those specific antigen variants that are predicted to be targeted by bactericidal antibodies elicited by the vaccine on different bacterial isolates.

Today, the most widely used approach for characterizing single meningococci is multilocus sequence typing (MLST), which defines strains from the sequences of seven housekeeping genes, including $\operatorname{arcC}$ (carbamate kinase), aroE (shikimate dehydrogenase), $g l p F$ (glycerol kinase), gmk (guanylate kinase), pta (phosphate acetyltransferase), tpi (triosephosphate isomerase), and yqiL (acetyl coenzyme A acetyltransferase) $[21,22]$. It should, however, be noted that the classification of strains based on MLST does not give direct indication on the $4 \mathrm{CMenB}$ antigenic repertoire. A study by Bambini et al. [18] demonstrated that each MLST clonal complex has an almost specific antigen variant repertoire resulting in a weak correlation between MLST and antigenic variability. It has been confirmed that the clonal complex alone generally has no discriminatory power to predict which strain will be killed on hSBA. These considerations make MLST only partially suitable for determining the phenotype profile, predicting vaccine antigen diversity, and, thus, assessing potential strain coverage.

Another potentially useful method to predict strain coverage is flow cytometry, which uses arrays of mono- and polyclonal antibodies and enables a considerable number of strains to be analyzed; currently, however, the method is implemented in few laboratories and may have standardization difficulties and, by using monoclonal antibodies only, it gives indication on the amount of antigens on the surface but not on their sequence diversity $[5,23]$.
To overcome the aforementioned limitations, a novel approach, termed the meningococcal antigen typing system (MATS), has been developed [14], its main aim being to predict the coverage of individual MenB strains provided by vaccination with $4 \mathrm{CMenB}$ by measuring the amount of antigen and its cross-reactivity. At the same time, since most capsular strains of $N$. meningitidis may express the same protein antigens $[24,25]$, the application of MATS could be extended to other serogroups. Moreover, this technique could be potentially adapted to other bacterial pathogens [26]. For these reasons recent advances and applications of MATS in the field of epidemiologic surveillance of bacteria should now be reviewed.

\section{MATS Development and Interpretation}

2.1. MATS as a Qualitative and Quantitative Assay. MATS was designed as a rapid and robust binding assay able to predict the susceptibility of individual MenB strains to be killed by bactericidal antibodies elicited by $4 \mathrm{CMenB}$; this method enables both qualitative (level of sequence relatedness) and quantitative (level of expression) evaluation of the antigens expressed on the surface of single strains [14, 15]. Both quantitative and qualitative aspects are highly important and should be assessed for the reasons described below.

The density and spatial orientation of an antigen on the bacterial surface are critical in the process of classical pathway of complement activation, which is initiated when a sufficient density of antigen-antibody complexes allows proximate fragment crystallizable $(\mathrm{Fc})$ regions of the antibody to bind Clq. An increase in surface antigen density results in a reduced distance between bound antibodies, thus leading to a higher probability of engagement and activation of the complement system $[27,28]$. On the other hand, the level of surface antigen expression is not the only factor that influences killing; the quality of the fit antigen-antibody is also crucial [29].

Basically, MATS is a modified sandwich enzyme-linked immunosorbent assay (ELISA) that quantifies expression and the level of matching with the corresponding antigen in the vaccine (fHbp, NHBA, and NadA) on bacterial lysates. Moreover, the PorA serosubtype is identified by means of the traditional PCR genotypic approach by assessing its variable region 2, and an individual strain matching for PorA (PorA 1.4) is considered to be covered by $4 \mathrm{CMenB}$. This subsequently enables antigenic cross-reactivity with the main corresponding $4 \mathrm{CMenB}$ components to be measured $[14,15]$.

Methodologically, the MATS ELISA procedure comprises several steps, which can be schematized as follows (see Figure 2). At the first step, cultures are grown overnight on chocolate agar plates at $37^{\circ} \mathrm{C}, 95 \%$ relative humidity, and $\mathrm{CO}_{2}$ concentration of $5 \%$. Subsequently, bacteria are suspended in Mueller-Hinton broth to achieve an optical density (OD) at $600 \mathrm{~nm}$ of 0.4 and then lysed with the detergent Empigen $\mathrm{BB} 5 \%$ added to a final volume of $1: 11$ $(0.45 \%)$ and inactivated at $45^{\circ} \mathrm{C}$ for 1 hour in a water bath. Twofold serial dilutions of the bacterial lysates are incubated in duplicate in three different ELISA microwell plates coated 


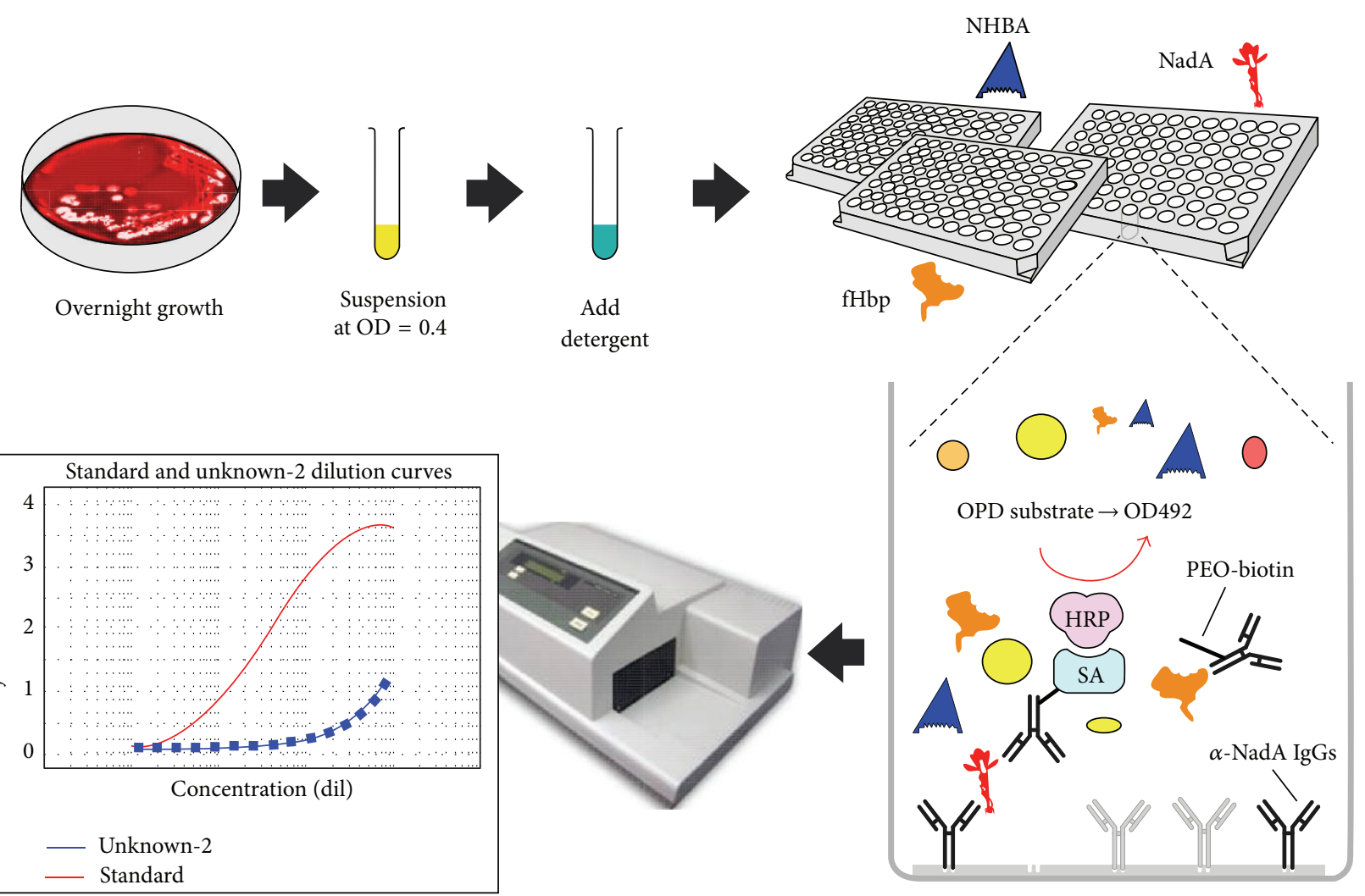

Data reduction

Read at $492 \mathrm{~nm}$

FIGURE 2: Schematic representation of the MATS ELISA method [15]. Bacteria from overnight cultures on agar chocolate plates are suspended in Mueller-Hinton broth and lysed with a detergent (Empigen BB 5\%) added to a final volume of 1/11 and inactivated for 1 hour at $45^{\circ} \mathrm{C}$ in a water bath; bacterial lysates are added to three different ELISA microwell plates coated with polyclonal rabbit antibodies raised against the single vaccine components fHbp, NHBA, and NadA; the antigens are captured from the suspension to the plate. Plates are then incubated for 1 hour at $37^{\circ} \mathrm{C}$ with biotinylated rabbit polyclonal antibodies against each of the antigens, washed, incubated with streptavidin-HRP, and developed with the OPD substrate. Relative potency is calculated by interpolating the regression curve of the unknown sample versus that of a reference strain added to each plate. Adapted with permission from [15].

with rabbit polyclonal antibodies against fHbp, NHBA, and NadA, respectively. The plates are incubated for 1 hour at $37^{\circ} \mathrm{C}$ and washed with a solution of phosphate buffered saline (PBS1x) plus $0.05 \%$ Tween. The amount of antigen bound to the antibodies is detected by incubating for 1 hour at $37^{\circ} \mathrm{C}$ with purified rabbit immunoglobulin $\mathrm{G}$ (IgG) raised against each of the three recombinant proteins, labeled with biotin. The plates are then washed and incubated for $30 \mathrm{~min}$ at $37^{\circ} \mathrm{C}$ with streptavidin-horseradish peroxidase and for $20 \mathrm{~min}$ at room temperature with the ortho-phenylene diamine substrate; the reaction is stopped by adding $50 \mu \mathrm{L}$ of sulfuric acid solution $(4 \mathrm{~N})$. Immediately afterwards, the plates are read at $492 \mathrm{~nm}$ by means of an ELISA reader $[14,15]$.

The MATS ELISA readout expresses the relative potency (RP) for fHbp, NHBA, and NadA of single strains; RPs of tested strains are calculated by means of a variance-weighted regression method by comparing the serial dilution curves of tested strains with those of the appropriate reference strains for each of the three antigens. The reference strains are as follows: H44/76 for fHbp, NGH38 for NHBA, and 5/99 for $\mathrm{NadA}$; the RPs are calculated by assigning the arbitrary value of 1 (or $100 \%)$ to each reference strain. Subsequently, in order to determine cut-off values of RPs that would predict susceptibility on hSBA, MATS RPs have been related to hSBA of pooled serum from 13-month-old children immunized with $4 \mathrm{CMenB}$ at 2, 4, 6, and 12 months of age. The positive bactericidal threshold (PBT) has been defined as the MATS RP point estimate, above which the majority of strains are killed in hSBA. These RP values are 0.021 (2.1\%) for $\mathrm{fHbp}$, $0.294(29.4 \%)$ for NHBA, and 0.009 (0.9\%) for NadA [14]. In sum, the MATS phenotype is defined as follows: each of the four antigens can be either positive (when RP > PBT or PorA is P1.4) or negative (when RP $\leq$ PBT and PorA is not P1.4) so that the number of possible MATS phenotypes is $2^{4}=16$ $[14,30]$.

2.2. Impact of Antigen Sequence Variation on the Relative Potency Values (Qualitative Aspects of MATS). As described above, the level of sequence relatedness of an antigen in a given MenB strain to the corresponding antigen included in the vaccine is a determining factor. The main $4 \mathrm{CMenB}$ antigens, fHbp, NHBA, and NadA, have a substantial level 
of sequence variability [16-18]. For instance, fHbp has been divided into three variants, namely, 1,2 , and 3 , each of which may be further classified into subvariants. Sequence conservation within variants is high (91.6-100\%), while that between variants is approximately $63 \%[17,18]$. Other fHbp classifications based on two subfamilies [31] and nine modular groups [20] have also been described. Similarly, several peptides of NHBA and at least five NadA variants have been established [16]. 4CMenB recombinant proteins include fHbp subvariant 1.1, NHBA subvariant 2, and NadA variant 3 [24]. MATS RPs of single antigens in MenB strains are highly influenced by such complex genetic variations. In a panel of $124 \mathrm{MenB}$ isolates, selected to represent a broad range of MLST and PorA types from different geographic areas, the highest RPs of fHbp (46-140\%) have been found among strains classified within fHbp subvariant 1.1. Strains expressing other subvariants of the $\mathrm{fHbp}$ variant 1 have shown significantly lower RPs of 1.6-38\%, while those expressing fHbp variants 2 and 3 have displayed RPs below the lower limit of quantitation. Although all tested strains harbor the nhba gene, only $70 \%$ of them have RPs above the lower limit of quantitation and a 6.5-fold range in RPs has been observed (from $20 \%$ to $130 \%$ ). The nadA gene has been found in only one-third of isolates and less than 20\% showed RPs above the lower limit of quantitation, with a more than 1000 -fold range in RP values [14].

Analogous results have been reported from Canada, where all isolates expressing fHbp subvariant 1.1 showed RPs > PBT, while the proportions of strains predicted to be covered by $4 \mathrm{CMenB}$ were $33 \%, 85 \%$, and $95 \%$ for those expressing the more distant subvariants $1.13,1.15$, and 1.4, respectively. None of the fHbp variant 2 or 3 strains had RPs above the PBT for $\mathrm{fHbp}$ and would require expression of a different vaccine antigen (i.e., PorA, NHBA, and NadA) in order to be covered [32]. Among European MenB isolates, strains with different subvariants of fHbp-1 have generally shown RPs > PBT, while almost all strains with fHbp-2 or fHbp- 3 have had RPs close to $0 \%$ or, in any case, below the PBT. A high variation in RPs for different variants of NHBA has also been documented in three studies $[5,32,33]$.

2.3. Association between MATS and hSBA. To investigate the relationship between MATS RPs and bactericidal titers, 57 strains from the $124 \mathrm{MenB}$ panel were tested by means of hSBA using pooled infant serum from 13-month-old children immunized with $4 \mathrm{CMenB}$ at $2,4,6$, and 12 months of age. To assess the contribution of each antigen to the bactericidal activity, a subset of 5 strains for fHbp, 11 for NHBA, and 7 for NadA that were mismatched to the vaccine for PorA and had only one vaccine antigen RP above the MATS lower limit of quantitation was selected. In this subset of strains the correlations between hSBA titers and relative potencies were statistically significant. Spearman's correlation coefficients were as follows: $\rho=0.97$ ( $P=0.005)$ for fHbp; $\rho=0.75$ $(P=0.008)$ for NHBA; $\rho=0.81(P=0.027)$ for NadA. Eighty-nine percent (39/44) of strains with RPs above the PBT for at least one antigen have been seen to be killed on hSBA (titer of $\geq 1: 8$ or 4 -fold rise) with pooled sera from
TABLE 1: Positive bacterial threshold (PBT) values with 95\% CIs for three antigens [26].

\begin{tabular}{lcc}
\hline Antigen & Estimate $\%$ & $95 \%$ CI \\
\hline fHbp & 2.1 & $1.4-3.1$ \\
NHBA & 29.4 & $16.9-51.1$ \\
NadA & 0.9 & $0.4-1.9$ \\
\hline
\end{tabular}

13-month-old children immunized with four $4 \mathrm{CMenB}$ doses. When considering RPs above the PBT for the single antigens, the highest positive predictive value was for $\mathrm{fHbp}(100 \%$, $7 / 7)$, followed by NadA $(83 \%, 5 / 6)$ and NHBA $(82 \%, 9 / 11)$. The negative predictive value (proportion of strains with all four RPs $\leq$ PBT that are not killed on hSBA) was also high $(77 \%, n=13)$ [14]. Similar findings have been observed among adults who received three $4 \mathrm{CMenB}$ doses: within the 124-strain panel, 83 out of 91 (91\%) strains showing RPs above the PBT for one or more antigens were killed on hSBA with pooled sera. Positive predictive values increased as the number of antigens with RP > PBT increased (1 antigen: 85\%, $n=41 ; 2$ antigens: $94 \%, n=34 ; 3$ antigens: $100 \%, n=16$ ) [14].

The use of pooled immune sera, however, may not accurately predict how each of the sera that compose the pool would react against a tested strain. Indeed, it is possible that a few subjects with unusually high or low hSBA titers may impact on the overall pooled response or that the potential synergy between antibodies from different subjects might generate a bactericidal response which would not be achieved individually. However, a recent study [34] aiming at investigating the relationship between pooled and individual sera has shown that individual responses to $4 \mathrm{CMenB}$ are homogeneously distributed and that pooled hSBA titers reflect the arithmetic mean of the individual titers with good approximation.

2.4. Reliability and Reproducibility of MATS. Interlaboratory standardization of MATS has been also achieved by using 17 shared MenB strains [26]. The log-transformed RPs obtained from five laboratories showed excellent robustness at different temperatures of sample inactivation $\left(37^{\circ} \mathrm{C}\right.$ and $45^{\circ} \mathrm{C}$ ), with Lin's coefficient of accuracy being 0.999 (95\% CI: $0.997-1$ ) and Pearson's correlation coefficient being 0.993 (95\% CI: 0.988-0.995). Log-transformed RPs from seven laboratories displayed almost perfect accuracy and concordance, with the corresponding coefficients exceeding 0.99. Within-laboratory variations in RPs for individual antigens differed slightly, being highest for NadA and lowest for $\mathrm{fHbp}$; this variance, however, was not affected by systematic bias. Similarly, between-laboratory coefficients of variation were $7.85 \%$ for fHbp, $12.60 \%$ for NHBA, and $16.51 \%$ for NadA. Moreover, in this study empirical estimates of $95 \%$ CI of PBTs (Table 1) for each individual antigen were calculated as $10^{\lg (\mathrm{PBT}) \pm 1.96 \cdot \sigma}$. The authors proposed using $95 \%$ confidence limits to predict $4 \mathrm{CMenB}$ coverage, as these would account for between- and within-laboratory variances. Laboratory qualification criteria were also clearly described. 
TABLE 2: Relative contribution (\%) of all possible antigen combinations to MATS-predicted coverage in three countries [5, 30, 32, 33].

\begin{tabular}{lccc}
\hline Antigen (MATS phenotype) & $\begin{array}{c}\text { England and Wales } \\
(n=535)\end{array}$ & $\begin{array}{c}\text { Canada } \\
(n=157)\end{array}$ & $\begin{array}{c}\text { Greece } \\
(n=148)\end{array}$ \\
\hline fHbp+/NHBA-/NadA-/PorA- & 14.6 & 12.7 & 9.5 \\
fHbp-/NHBA+/NadA-/PorA- & 7.9 & 13.4 & 33.8 \\
fHbp-/NHBA-/NadA+/PorA- & - & - & 0.7 \\
fHbp-/NHBA-/NadA-/PorA+ & 0.4 & - & 0.7 \\
fHbp+/NHBA+/NadA-/PorA- & 29.9 & 25.5 & 37.1 \\
fHbp+/NHBA-/NadA+/PorA- & - & 0.6 & - \\
fHbp+/NHBA-/NadA-/PorA+ & 3.2 & 1.9 & - \\
fHbp-/NHBA+/NadA-/PorA+ & 0.9 & - & 1.3 \\
fHbp-/NHBA+/NadA+/PorA- & 0.4 & 0.6 & - \\
fHbp-/NHBA-/NadA+/PorA+ & - & - & - \\
fHbp+/NHBA+/NadA+/PorA- & 0.2 & - & - \\
fHbp+/NHBA+/NadA-/PorA+ & 15.7 & 11.5 & 6.1 \\
fHbp+/NHBA-/NadA+/PorA+ & - & - & - \\
fHbp-/NHBA+/NadA+/PorA+ & - & - & - \\
fHbp+/NHBA+/NadA+/PorA+ & - & - & - \\
fHbp-/NHBA-/NadA-/PorA- & 26.9 & 33.8 & 10.8 \\
\hline
\end{tabular}

\section{Field Applications of MATS}

3.1. Application of MATS to Estimate Country-Specific Serogroup B Strain Coverage of 4 CMenB. In a large study [5] (1,052 MenB isolates from five countries) carried out in Europe, the results of MATS predicted an overall strain coverage of $78 \%$ (95\% CI: 63-90\%), and half of the tested strains would have been covered by at least two vaccine antigens. The most common MATS phenotypes were fHbp plus NHBA, no antigen, fHbp plus NHBA plus PorA, fHbp, and NHBA; expression of NadA was above the positive bactericidal thresholds in only a small proportion [7\% (16/235)] of isolates that harboured the NadA gene. The robustness of estimates was further established by MATS testing of additional MenB isolates from another two countries (Spain and the Czech Republic). Some level of between-country variation in coverage estimates was observed: 87\% (95\% CI: 70-93\%) in Italy, 85\% (95\% CI: 7698\%) in Norway, 85\% (95\% CI: 69-93\%) in France, 82\% (95\% CI: 69-92\%) in Germany, 74\% (95\% CI: 58-87\%) in the Czech Republic, 73\% (95\% CI: 57-85\%) in England and Wales, and 69\% (95\% CI: 48-85\%) in Spain. However, this difference was not statistically significant at an $\alpha$ level of $<0.05$. A somewhat lower predicted strain coverage of $66 \%$ (95\% CI: $46-78 \%$ ) has been reported in Canada. As in the European study, most tested strains could have been targeted by bactericidal antibodies against $>1$ vaccine antigen. Thus, $29 \%$ and $11.5 \%$ of isolates were covered by two and three antigens, respectively. The highest relative contribution to coverage was made by fHbp and NHBA [32]. A more recent Greek study has revealed an MATS-predicted coverage of $89.2 \%$ (95\% CI: $63.5-98.6 \%$ ) by at least one antigen and $44.5 \%$ by at least two antigens. Again, NHBA and $\mathrm{fHbp}$ were the greatest contributors to coverage, while PorA and NadA contributed significantly less [33]. Table 2 reports the contribution of each
4CMenB antigen and their combinations to MATS-predicted coverage in three countries.

3.2. Application of MATS to Estimate Serogroup X Strain Coverage of $4 C M e n B$. Today, the $N$. meningitidis group $\mathrm{X}$ (MenX), which has caused several outbreaks in Africa, remains the only serogroup for which no vaccine exists. The cross serogroup immunogenicity elicited by 4 CMenB is biologically plausible, since the majority of capsular strains may express the vaccine antigens [24]. In a paper by Hong et al. [35], nine African MenX isolates and two French MenX isolates were tested by means of MATS in order to determine the presence, diversity, and expression level of $4 \mathrm{CMenB}$ antigens. In this study, the PBT values estimated for MenB isolates were applied to MenX strains; the strains were also analyzed by hSBA by using preimmunization and postimmunization sera from different age groups. All African isolates displayed MATS RP values for fHbp above PBT (2.5-5\%); conversely, the two French strains showed RP values below the lower limit of quantitation for fHbp, as a result of mismatching for $\mathrm{fHbp}$. Among the African isolates, RPs for NHBA ranged from $11.3 \%$ to $31.5 \%$; only three isolates displayed RP values above the PBT. Moreover, one African and one French isolate had the nadA gene, but its expression was very low. Although a correlation between MATS RP and hSBA has not yet been established for non-B strains, good agreement was observed between MATS RPs and killing in hSBA in all the age groups for the MenX strains tested in this study.

3.3. Application of MATS to Estimate the Potential Impact of $4 C M e n B$ on Carriage. The impact of meningococcal vaccination on asymptomatic nasopharyngeal carriage is of primary importance, since healthy carriers are the main reservoir of $N$. 
meningitidis [36]. Again, MATS may provide useful insights into carriage status, as has been documented in two recent studies [37, 38]. Claus et al. [37] applied MATS to investigate whether 41 capsule-null locus meningococci isolated from Germany, the Czech Republic, and Burkina Faso harbored the genes and expressed the main $4 \mathrm{CMenB}$ antigens. However, as PBT had not yet been assessed for capsule-null locus meningococci, PBT values were not applied in their study. Six strains belonging to the clonal complex ST-198 expressed fHbp subvariant 1.4, with RPs ranging from $7.9 \%$ to $11.2 \%$. NHBA was expressed in all 41 strains, with RP values of 2.3-58.2\%; the highest RPs were seen in isolates belonging to clonal complexes ST-198 and ST-845. The nadA gene was found in only one isolate; the expression of the NadA protein was below the limit of detection. No strain matched for the PorA included in 4CMenB. Interestingly, the authors documented that the presence of a capsule has almost no impact on antigen detection levels. In particular, MenB strains MC58 and H44/76 and their corresponding capsulenull isogenic mutants, as well as the capsule-null isolate $\alpha 30$ and its encapsulated derivative, showed very similar RPs for fHbp and NHBA [37].

In an Italian longitudinal carrier study [38] 32 out of 173 students tested positive for $N$. meningitidis of different serogroups in at least 1 of 4 examinations. As shown by MATS, strains expressing subvariants of $\mathrm{fHbp}-1$ isolated from 5 students showed detectable expression levels of the antigen, with RPs of 1.2-122.1\%. Conversely, isolates carrying fHbp-2 and $\mathrm{fHbp}-3$ were negative on MATS. NHBA was detectable in all isolates (RPs of 2.5-131.2\%), while, as expected, NadA expression was detected in only one subject, being NadA very poorly expressed in carrier strains. This study also revealed that RPs for individual antigens remained comparable in subjects on consecutive positive swabs, with the exception of NadA, for which the only positive subject showed a more than 10 -fold higher RP on the second swab (43\%) than on the first, third, and fourth $(3.7 \%, 3.4 \%$, and $3.1 \%$, resp.).

These two studies $[37,38]$ have indicated that $4 \mathrm{CMenB}$ will very probably affect the carriage and acquisition of $N$. meningitidis; the impact on the unencapsulated strains will probably be less pronounced. It must, however, be borne in mind that, in the case of capsule-null locus strains, the association between hSBA and MATS is quite difficult to establish, owing to their intrinsic susceptibility to complementmediated killing [38]. In any case, vaccine-induced changes in circulating meningococcal strains and detailed typing of both invasive and carrier isolates should be subject to strict monitoring in the near future.

\section{MATS May Underestimate 4CMenB Coverage}

Intrinsically, MATS is a conservative predictor of MenB strain coverage; indeed, in comparison with hSBA, it underestimates coverage. Frosi et al. [30] applied a stratified proportional random sampling procedure to select a representative panel of $40 \mathrm{MenB}$ isolates from the overall panel of 535 isolates collected in England and Wales over 2007-2008.
The selected isolates were tested in the hSBA assay with pooled sera from infant and adolescent vaccinees, and the results compared by means of MATS. In this study, MATSbased predictions of coverage of 70\% (95\% CI: 55-85\%) were largely confirmed by $88 \%$ killing in hSBA (95\% CI: 72-95\%); 27 true positives and 4 true negatives were found, yielding overall accuracy of $78 \%$; positive and negative predictive values were $96 \%$ and $33 \%$, respectively [30]. Thus, coverage predicted by MATS has been largely confirmed by subsequent hSBA on pooled infant and adolescent postvaccination sera. This discrepancy between MATS and hSBA assessments of coverage could be explained by various factors.

4.1. Non-PorA Components of OMV. One of the four main 4CMenB components is OMV from MenB strain NZ98/254 [39]. OMVs are released by several bacterial species and contain various outer membrane proteins, lipopolysaccharide, and a lumen with periplasmic constituents [40]. Apart from PorA, other proteins identified in the outer membrane have been shown to be immunogenic, including, for example, opacity-associated proteins (Opc and Opa) [41] and Neisseria surface protein A (NspA) [42]. MATS ELISA does not detect antibodies elicited against such OMV components.

4.2. Synergistic or Additive Effects of Antibodies against Multiple Antigens. MATS does not consider the possible effects of bactericidal antibodies against more than one antigen present in $4 \mathrm{CMenB}$, including different outer membrane proteins. Giuliani et al. [43] have documented that antibodies against non-PorA antigens present in OMV, which may be either bactericidal or not, are able to induce a synergistic bactericidal activity with antibodies against fHbp. Similarly, anti-NHBA antibodies may exert a cooperative activity with antibodies against other antigens [44]. Sera from six subjects immunized with a meningococcal vaccine containing recombinant GNA2091-fHbp, NHBA-GNA1030, and NadA were tested in hSBA both before and after depletion of antifHbp and/or anti-NHBA antibodies. All vaccinees showed at least a 4 -fold increase in hSBA titers in comparison with their preimmune serum against the test strain $\mathrm{H} 44 / 76$, which matched the fHbp present in the vaccine. When anti-fHbp antibodies were depleted, hSBA titers decreased by at least $88 \%$ in all subjects. Four of six vaccinees showed at least a 4-fold increase in hSBA titers against the strain M4407, which expresses a heterologous to the vaccine $\mathrm{fHbp}-2$ and a homologous to the vaccine NHBA amino acid sequence. In one vaccinee, hSBA was directed mostly against NHBA, in another vaccinee against $\mathrm{fHbp}$, while in the remaining two subjects depletion of antibodies against either fHbp or NHBA more than halved the hSBA titer. In all four subjects, depletion of both anti-fHbp and anti-NHBA antibodies suppressed bactericidal activity to a greater degree than depletion of these antibodies individually, indicating a cooperative bactericidal activity between antibodies against fHbp and NHBA. The same study showed that, in mice immunized with only fHbp variant 1 antigen or NHBA vaccine antigen, the percentage survival of strain M4407 incubated with a 1:1 mixture of pooled sera from mice immunized with NHBA and pooled 
sera from mice immunized with fHbp was lower than that when the strain was incubated with a 1:1 mixture of pooled sera from mice immunized with NHBA and pooled sera from negative control mice immunized with aluminum hydroxide [44].

4.3. NadA In Vitro Downregulation. In comparison with both NHBA and fHbp, the relative contribution of NadA to $4 \mathrm{CMenB}$ coverage has proved to be very low in all studies conducted so far $[5,14,32,33]$, despite the fact that this antigen is carried by approximately one-third of pathogenic isolates and by three of four hypervirulent lineages. The most probable reason for this observation is the very complex mechanisms of nadA gene regulation. The phase-variable expression of nadA is mostly mediated by NadR, which represses nadA [45]. Indeed, under in vitro conditions in both hSBA and MATS, nadA repression by NadR results in inefficient killing of various MenB strains by anti-NadA antibodies. However, it has been suggested that nadA expression in vitro may differ from that in vivo. In this regard, sera from children infected with strains that do not express NadA $(\mathrm{RP}=0 \%)$ or displaying low NadA expression $(\mathrm{RP}<\mathrm{PBT})$ have been seen to recognize NadA recombinant proteins, also confirming the hypothesis that $\mathrm{NadA}$ is less expressed in vitro; this degree of recognition is much higher than that observed in sera from subjects infected by nadA negative strains [46]. Knocking out $n a d R$ results in a higher level of NadA expression and efficient killing by sera from subjects vaccinated with 4CMenB. Moreover, the MenB strain NGP165, which is mismatched for fHbp, NHBA, and PorA and has NadA $\mathrm{RP}<\mathrm{PBT}$, is not killed by sera from infants vaccinated with $4 \mathrm{CMenB}$ when it is grown in vitro. However, the same infant sera provide passive in vivo protection against NGP165 bacteremia, as has been shown in an infant rat model [47].

\section{Conclusion}

This paper attempts to critically review available studies that have used MATS. Implementation of MATS is expected to grow steadily, as $4 \mathrm{CMenB}$ has recently been approved for human use in several countries [48-50]. MATS could be useful in tracking spatial and temporal changes in MenB epidemiology and their repercussions on $4 \mathrm{CMenB}$ coverage. As anticipated by Vogel et al. [23], there are two main reasons for the increased use of MATS as follows: (i) implementation of the vaccine will potentially affect the population structure of $N$. meningitidis, in that the proportion of strains not covered by $4 \mathrm{CMenB}$ will probably increase, thus requiring additional surveillance efforts and (ii) careful assessment of vaccination failures.

A possible limitation of this method could be the lack of information on the relationship between pooled sera, which are used to calculate PBT, and the response rate of individual subjects; this aspect has not yet been fully investigated and is currently under evaluation.

Although MATS has been established for MenB strains, the method is flexible enough to be modified for application to other N. meningitidis serogroups and bacteria species. To date, PBT values have been established only for serogroup $\mathrm{B}$, thereby limiting MATS application to this serogroup. However, as the genetic diversity of other serogroups is substantially lower, the establishment of PBTs for these should require a lower number of strains. Moreover, reverse vaccinology is being applied to a number of other bacteria [51], and MATS ELISA could be adapted in order to assess these pathogens [26].

Across the studies conducted so far $[5,32,33]$, the MATSpredicted MenB strain coverage provided by $4 \mathrm{CMenB}$ has shown some differences among areas or countries, with minimum values observed in Canada and maximum values in Greece. Apart from the diversity in circulating strains, a possible explanation for this variability is that MenB strain panels may not be fully representative, since they generally come from passive surveillance systems [5]. Likewise, it must be taken into account that MATS underestimates the vaccine coverage [30, 41, 46, 47].

Accurate data on the potential strain coverage provided by the new multicomponent vaccine are of crucial interest to policy-makers in order to decide whether to introduce the vaccine into national immunization programs. MenB coverage estimates based on MATS have already been used in various economic evaluations of $4 \mathrm{CMenB}[52,53]$.

In conclusion, MATS is an innovative technique that has several advantages, including (but not limited to) the following:

(i) assessment of both the level of expression and antigenic relatedness of fHbp, NHBA, and NadA vaccine antigens in meningococci isolates;

(ii) from the technical standpoint, MATS is more rapid and resource-saving than other approaches;

(iii) despite some level of underestimation, MATS strongly correlates with the universally accepted correlate of protection and provides satisfactorily accurate MenB coverage predictions;

(iv) it is highly reproducible and can be successfully transferred to new laboratories;

(v) it enables near real-time estimation of the postimplementation effectiveness of $4 \mathrm{CMenB}$;

(vi) $\mathrm{PBT}$ values may be used to efficiently predict $4 \mathrm{CMenB}$ strain coverage on a representative panel of invasive isolates from any geographical setting; such estimates are crucial to economic and health technology assessment (HTA) studies.

\section{Conflict of Interests}

Giuseppe Boccadifuoco and Marzia Monica Giuliani are employees of Novartis Vaccines. Alexander Domnich, Roberto Gasparini, Daniela Amicizia, and Donatella Panatto declare that there is no conflict of interests regarding the publication of this paper. 


\section{References}

[1] R. Z. Jafri, A. Ali, N. E. Messonnier et al., "Global epidemiology of invasive meningococcal disease," Population Health Metrics, vol. 11, no. 1, article 17, 2013.

[2] D. Panatto, D. Amicizia, P. L. Lai, M. L. Cristina, A. Domnich, and R. Gasparini, "New versus old meningococcal Group B vaccines: how the new ones may benefit infants \& toddlers," Indian Journal of Medical Research, vol. 138, pp. 835-846, 2013.

[3] World Health Organization (WHO), "Meningococcal vaccines: WHO position paper, November 2011," Weekly Epidemiological Record, vol. 86, no. 47, pp. 521-539, 2011.

[4] R. Rappuoli, "Reverse vaccinology, a genome-based approach to vaccine development," Vaccine, vol. 19, no. 17-19, pp. 2688-2691, 2001.

[5] U. Vogel, M.-K. Taha, J. A. Vazquez et al., "Predicted strain coverage of a meningococcal multicomponent vaccine (4CMenB) in Europe: a qualitative and quantitative assessment," The Lancet Infectious Diseases, vol. 13, no. 5, pp. 416-425, 2013.

[6] M. M. Giuliani, J. Adu-Bobie, M. Comanducci et al., "A universal vaccine for serogroup B meningococcus," Proceedings of the National Academy of Sciences of the United States of America, vol. 103, no. 29, pp. 10834-10839, 2006.

[7] World Health Organization (WHO), Correlates of VaccineInduced Protection: Methods and Implications, World Health Organization (WHO), Geneva, Switzerland, 2013.

[8] S. A. Plotkin and P. B. Gilbert, "Nomenclature for immune correlates of protection after vaccination," Clinical Infectious Diseases, vol. 54, no. 11, pp. 1615-1617, 2012.

[9] A. C. Conn, J. R. MacNeil, L. H. Harrison et al., "Changes in neisseria meningitidis disease epidemiology in the united states, 1998-2007: implications for prevention of meningococcal disease," Clinical Infectious Diseases, vol. 50, no. 2, pp. 184-191, 2010.

[10] European Centre for Disease Prevention and Control (ECDC), Surveillance of Invasive Bacterial Diseases in Europe, 2011, European Centre for Disease Prevention and Control, Stockholm, Sweden, 2013.

[11] L. Qin, P. B. Gilbert, L. Corey, M. J. McElrath, and S. G. Self, "A framework for assessing immunological correlates of protection in vaccine trials," Journal of Infectious Diseases, vol. 196, no. 9, pp. 1304-1312, 2007.

[12] C. E. Frasch, R. Borrow, and J. Donnelly, "Bactericidal antibody is the immunologic surrogate of protection against meningococcal disease," Vaccine, vol. 27, supplement 2, pp. B112-B116, 2009.

[13] R. Borrow, G. M. Carlone, N. Rosenstein et al., "Neisseria meningitidis group B correlates of protection and assay standardization-international meeting report Emory University, Atlanta, Georgia, United States, 16-17 March 2005," Vaccine, vol. 24, no. 24, pp. 5093-5107, 2006.

[14] J. Donnelly, D. Medinia, G. Boccadifuoco et al., "Qualitative and quantitative assessment of meningococcal antigens to evaluate the potential strain coverage of protein-based vaccines," Proceedings of the National Academy of Sciences of the United States of America, vol. 107, no. 45, pp. 19490-19495, 2010.

[15] G. Boccadifuoco, B. Brunelli, M. G. Pizza, and M. M. Giuliani, "A combined approach to assess the potential coverage of a multicomponent protein-based vaccine," Journal of Preventive Medicine and Hygiene, vol. 53, no. 2, pp. 56-60, 2012.

[16] J. Lucidarme, M. Comanducci, J. Findlow et al., "Characterization of fHbp, nhba (gna2132), nadA, porA, and sequence type in group B meningococcal case isolates collected in England and Wales during January 2008 and potential coverage of an investigational group B meningococcal vaccine," Clinical and Vaccine Immunology, vol. 17, no. 6, pp. 919-929, 2010.

[17] V. Masignani, M. Comanducci, M. M. Giuliani et al., "Vaccination against Neisseria meningitidis using three variants of the lipoprotein GNA1870," Journal of Experimental Medicine, vol. 197, no. 6, pp. 789-799, 2003.

[18] S. Bambini, A. Muzzi, P. Olcen, R. Rappuoli, M. Pizza, and M. Comanducci, "Distribution and genetic variability of three vaccine components in a panel of strains representative of the diversity of serogroup B meningococcus," Vaccine, vol. 27, no. 21, pp. 2794-2803, 2009.

[19] D. M. Vu, R. Pajon, D. C. Reason, and D. M. Granoff, "A broadly cross-reactive monoclonal antibody against an epitope on the $\mathrm{N}$-terminus of meningococcal fHbp," Scientific Reports, vol. 2, article 341, 2012.

[20] P. T. Beemink and D. M. Granoff, "The modular architecture of meningococcal factor H-binding protein," Microbiology, vol. 155, no. 9, pp. 2873-2883, 2009.

[21] M. C. J. Maiden, J. A. Bygraves, E. Feil et al., "Multilocus sequence typing: a portable approach to the identification of clones within populations of pathogenic microorganisms," Proceedings of the National Academy of Sciences of the United States of America, vol. 95, no. 6, pp. 3140-3145, 1998.

[22] D. M. Aanensen and B. G. Spratt, "The multilocus sequence typing network: mlst.net," Nucleic Acids Research, vol. 33, supplement 2, pp. W728-W733, 2005.

[23] U. Vogel, P. Stefanelli, J. Vazquez, M.-K. Taha, H. Claus, and J. Donnelly, "The use of vaccine antigen characterization, for example by MATS, to guide the introduction of meningococcus B vaccines," Vaccine, vol. 30, no. 2, pp. B73-B77, 2012.

[24] R. Gasparini, D. Amicizia, A. Domnich, P. L. Lai, and D. Panatto, "Neisseria meningitidis B vaccines: recent advances and possible immunization policies," Expert Review of Vaccines, vol. 13, no. 3 , pp. 345-364, 2014.

[25] S. L. Harris, D. Zhu, E. Murphy et al., "Preclinical evidence for the potential of a bivalent fHBP vaccine to prevent Neisseria meningitidis serogroup C disease," Human Vaccines, vol. 7, supplement 1, pp. 68-74, 2011.

[26] B. D. Plikaytis, M. Stella, G. Boccadifuoco et al., "Interlaboratory standardization of the sandwich enzyme-linked immunosorbent assay designed for MATS, a rapid, reproducible method for estimating the strain coverage of investigational vaccines," Clinical and Vaccine Immunology, vol. 19, no. 10, pp. 1609-1617, 2012.

[27] J. A. Welsch, S. Ram, O. Koeberling, and D. M. Granoff, "Complement-dependent synergistic bactericidal activity of antibodies against factor $\mathrm{H}$-binding protein, a sparsely distributed meningococcal vaccine antigen," The Journal of Infectious Diseases, vol. 197, no. 7, pp. 1053-1061, 2008.

[28] V. E. Weynants, C. M. Feron, K. K. Goraj et al., "Additive and synergistic bactericidal activity of antibodies directed against minor outer membrane proteins of Neisseria meningitidis," Infection and Immunity, vol. 75, no. 11, pp. 5434-5442, 2007.

[29] B. Brunelli, E. Del Tordello, E. Palumbo et al., "Influence of sequence variability on bactericidal activity sera induced by Factor H binding protein variant 1.1," Vaccine, vol. 29, no. 5, pp. 1072-1081, 2011.

[30] G. Frosi, A. Biolchi, M. L. Sapio et al., "Bactericidal antibody against a representative epidemiological meningococcal serogroup B panel confirms that MATS underestimates 
4CMenB vaccine strain coverage," Vaccine, vol. 31, no. 43, pp. 4968-4974, 2013.

[31] L. D. Fletcher, L. Bernfield, V. Barniak et al., "Vaccine potential of the Neisseria meningitidis 2086 lipoprotein," Infection and Immunity, vol. 72, no. 4, pp. 2088-2100, 2004.

[32] J. A. Bettinger, D. W. Scheifele, S. A. Halperin et al., "Diversity of Canadian meningococcal serogroup B isolates and estimated coverage by an investigational meningococcal serogroup $\mathrm{B}$ vaccine (4CMenB)," Vaccine, vol. 32, no. 1, pp. 124-130, 2013.

[33] G. Tzanakaki, E. Hong, K. Kesanopoulos et al., "Diversity of greek meningococcal serogroup B isolates and estimated coverage of the $4 \mathrm{CMenB}$ meningococcal vaccine," BMC Microbiology, vol. 14, no. 1, article 111, 2014.

[34] S. Budroni, A. Kleinschmidt, E. Ypma, P. Boucher, and D. Medini, "Pooled hSBA titers predict seroresponse rates of infants vaccinated with 4CMenB," in Proceedings of the 19th International Pathogenic Neisseria Conference (IPNC '14), Asheville, NC, USA, October 2014.

[35] E. Hong, M. M. Giuliani, A.-E. Deghmane et al., "Could the multicomponent meningococcal serogroup $\mathrm{B}$ vaccine (4CMenB) control Neisseria meningitidis capsular group X outbreaks in Africa?" Vaccine, vol. 31, no. 7, pp. 1113-1116, 2013.

[36] H. Christensen, M. May, L. Bowen, M. Hickman, and C. L. Trotter, "Meningococcal carriage by age: a systematic review and meta-analysis," The Lancet Infectious Diseases, vol. 10, no. 12, pp. 853-861, 2010.

[37] H. Claus, M. S. Jördens, P. Kriz et al., "Capsule null locus meningococci: typing of antigens used in an investigational multicomponent meningococcus serogroup B vaccine," Vaccine, vol. 30, no. 2, pp. 155-160, 2012.

[38] R. Gasparini, M. Comanducci, D. Amicizia et al., "Molecular and serological diversity of Neisseria meningitidis carrier strains isolated from Italian students aged 14 to 22 years," Journal of Clinical Microbiology, vol. 52, no. 6, pp. 1901-1910, 2014.

[39] European Medicines Agency (EMA), "Bexsero: summary of product characteristics," http://www.ema.europa.eu/docs/en_ GB/document_library/EPAR_-_Product_Information/human/ 002333/WC500137881.pdf.

[40] B. van de Waterbeemd, G. Zomer, J. van den Ijssel et al., "Cysteine depletion causes oxidative stress and triggers outer membrane vesicle release by Neisseria meningitidis; implications for vaccine development," PLoS ONE, vol. 8, no. 1, Article ID e54314, 2013.

[41] E. Rosenqvist, E. A. Hoiby, E. Wedege, B. Kusecek, and M. Achtman, "The $5 \mathrm{C}$ protein of Neisseria meningitidis is highly immunogenic in humans and induces bactericidal antibodies," The Journal of Infectious Diseases, vol. 167, no. 5, pp. 1065-1073, 1993.

[42] N. Cadieux, M. Plante, C. R. Rioux, J. Hamel, B. R. Brodeur, and D. Martin, "Bactericidal and cross-protective activities of a monoclonal antibody directed against Neisseria meningitidis NspA outer membrane protein," Infection and Immunity, vol. 67, no. 9, pp. 4955-4959, 1999.

[43] M. M. Giuliani, A. Biolchi, D. Serruto et al., "Measuring antigenspecific bactericidal responses to a multicomponent vaccine against serogroup B meningococcus," Vaccine, vol. 28, no. 31, pp. 5023-5030, 2010.

[44] D. M. Vu, T. T. Wong, and D. M. Granoff, "Cooperative serum bactericidal activity between human antibodies to meningococcal factor $\mathrm{H}$ binding protein and Neisserial heparin binding antigen," Vaccine, vol. 29, no. 10, pp. 1968-1973, 2011.
[45] M. M. E. Metruccio, E. Pigozzi, D. Roncarati et al., "A novel phase variation mechanism in the meningococcus driven by a ligand-responsive repressor and differential spacing of distal promoter elements," PLoS Pathogens, vol. 5, no. 12, Article ID e1000710, 2009.

[46] D. J. Litt, S. Savino, A. Beddek et al., "Putative vaccine antigens from Neisseria meningitidis recognized by serum antibodies of young children convalescing after meningococcal disease," The Journal of Infectious Diseases, vol. 190, no. 8, pp. 1488-1497, 2004.

[47] L. Fagnocchi, A. Biolchi, F. Ferlicca et al., "Transcriptional regulation of the nada gene in neisseria meningitidis impacts the prediction of coverage of a multicomponent meningococcal serogroup b vaccine," Infection and Immunity, vol. 81, no. 2, pp. 560-569, 2013.

[48] European Medicines Agency (EMA), Bexsero Authorization Details, European Medicines Agency, London, UK, 2013, http:// www.ema.europa.eu/ema/index.jsp?curl=pages/medicines/human/medicines/002333/human_med_001614.jsp\&mid=WC0b$01 \mathrm{ac} 058001 \mathrm{~d} 124$.

[49] Australian Technical Advisory Group on Immunisation (ATAGI), "Advice for immunisation providers regarding the use of Bexsero," http://www.immunise.health.gov.au/internet/immunise/publishing.nsf/Content/atagi-advice-bexsero.

[50] Health Canada, Bexsero Summary Basis of Decision, Health Canada, 2014, http://www.hc-sc.gc.ca/dhp-mps/prodpharma/ sbd-smd/drug-med/sbd_smd_2014_bexsero_147275-eng.php.

[51] C. Donati and R. Rappuoli, "Reverse vaccinology in the 21st century: improvements over the original design," Annals of the New York Academy of Sciences, vol. 1285, no. 1, pp. 115-132, 2013.

[52] S. Capri, M. A. Veneziano, and C. de Waure, "Valutazione economica di Bexsero," Quaderni dell'Italian Journal of Public Health, vol. 2, no. 13, pp. 68-79, 2013.

[53] H. A. T. Tu, S. L. Deeks, S. K. Morris et al., "Economic evaluation of meningococcal serogroup B childhood vaccination in Ontario, Canada," Vaccine, vol. 32, no. 42, pp. 5436-5446, 2014. 


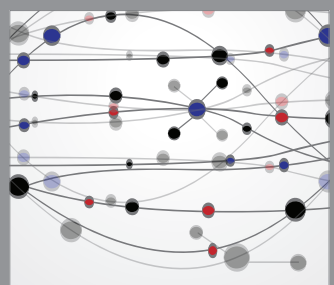

The Scientific World Journal
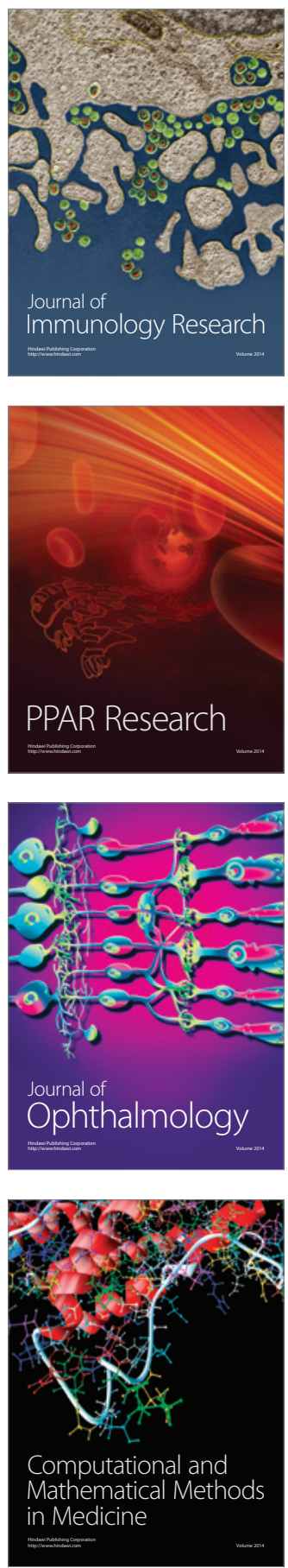

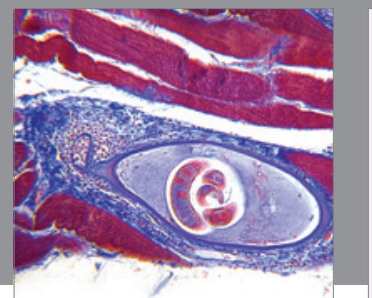

Gastroenterology

Research and Practice
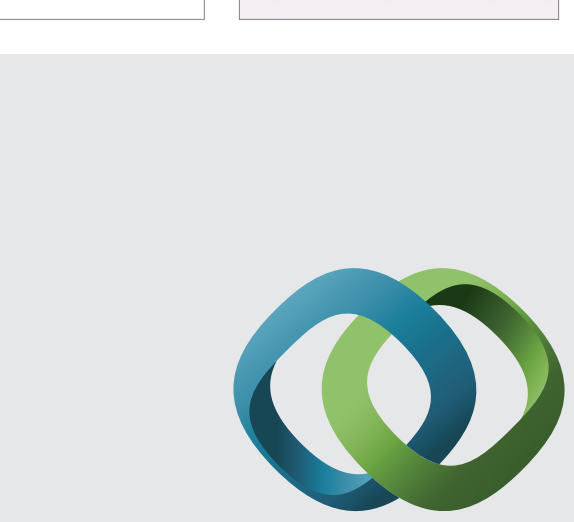

\section{Hindawi}

Submit your manuscripts at

http://www.hindawi.com
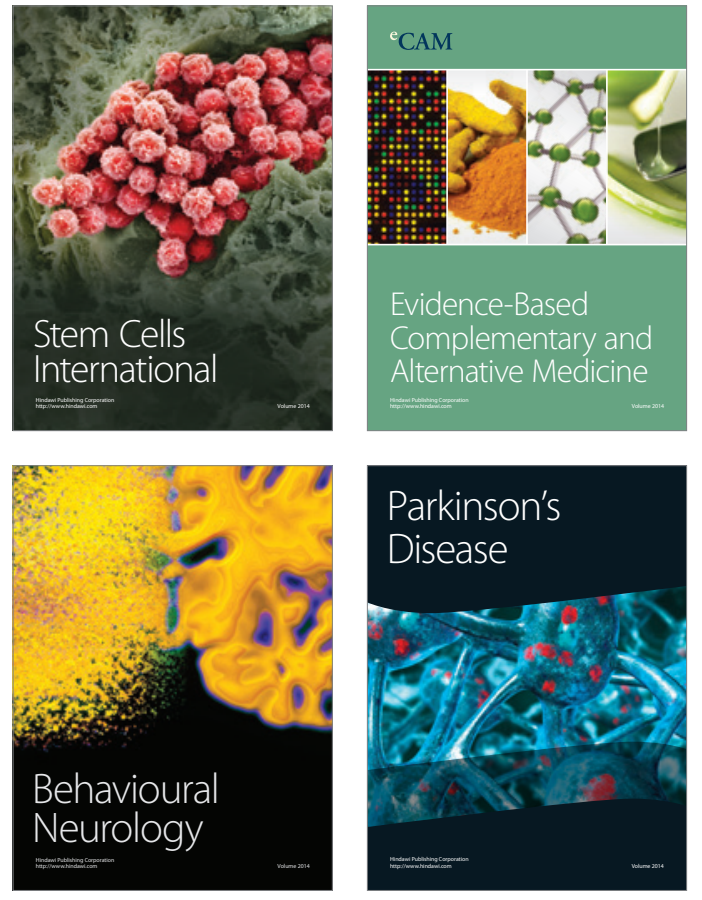
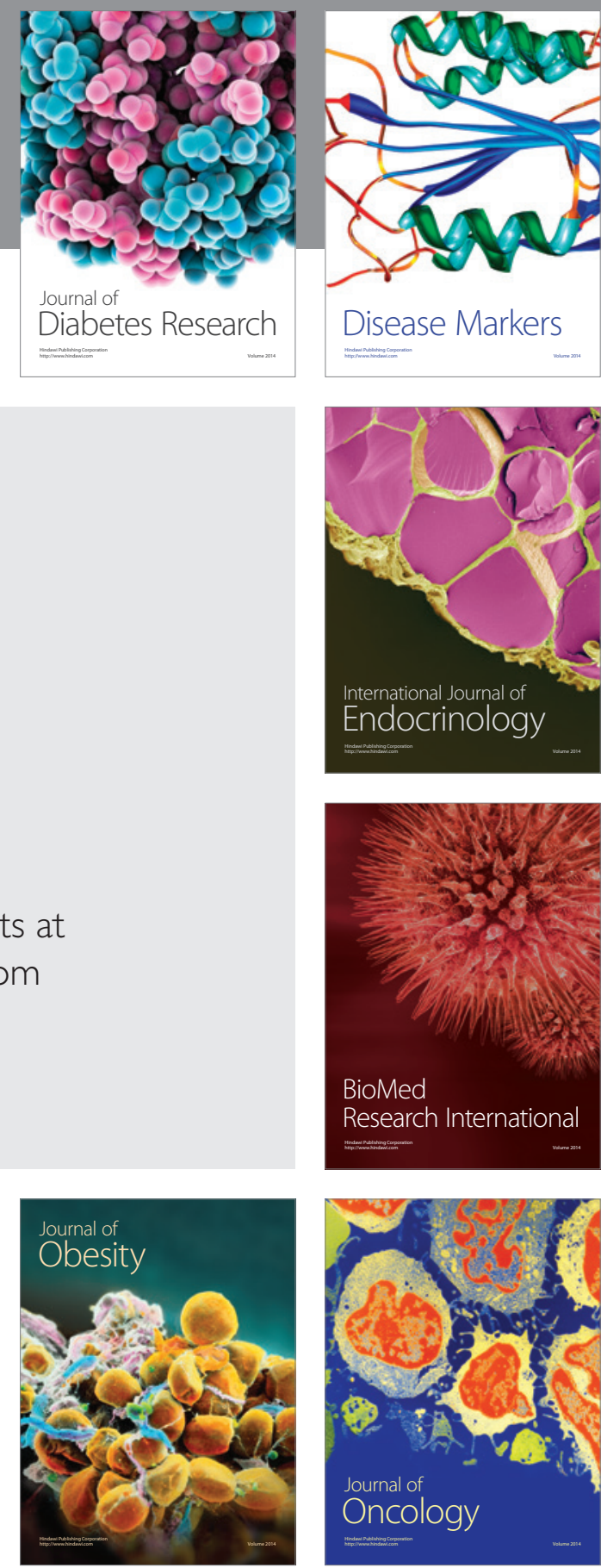

Disease Markers
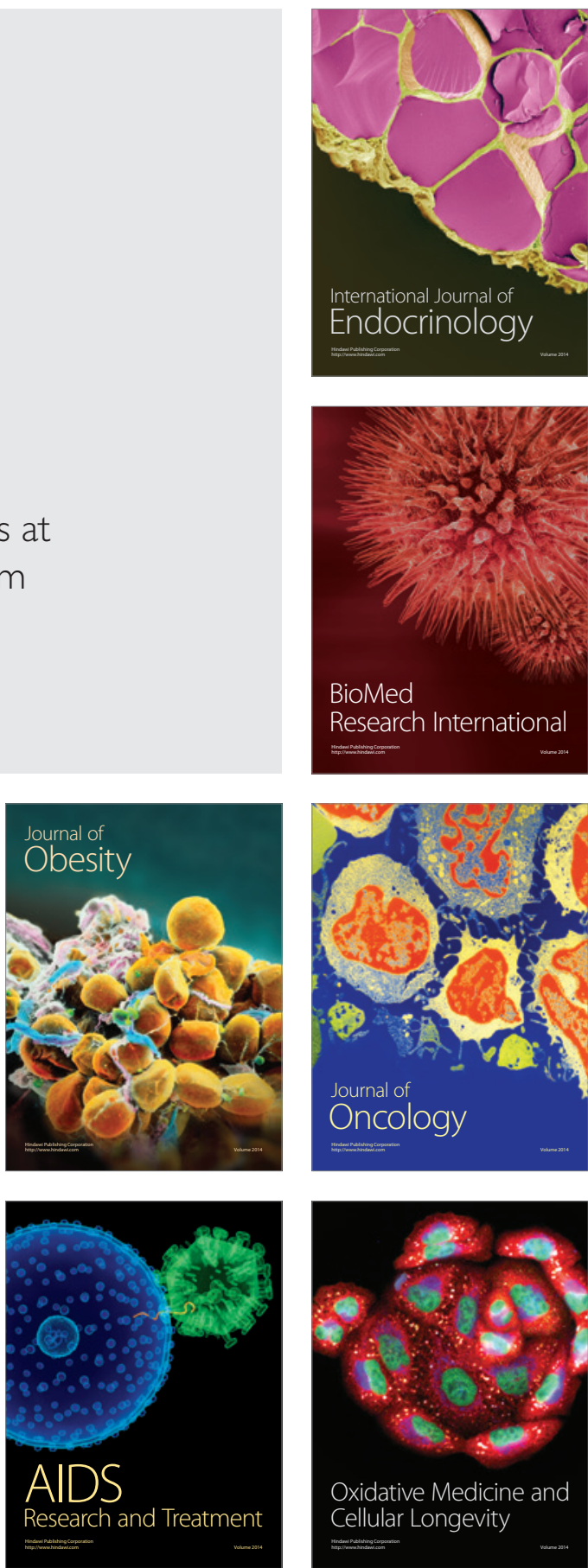